\title{
Article \\ Effects of Betahistine on the Development of Vestibular Compensation after Unilateral Labyrinthectomy in Rats
}

\author{
Junya Fukuda ${ }^{1, *(\mathbb{C})}$, Kazunori Matsuda ${ }^{2}$, Go Sato ${ }^{1}$, Tadashi Kitahara ${ }^{3}{ }^{\mathbb{C}}$, Momoyo Matsuoka ${ }^{1}$, Takahiro Azuma ${ }^{1}$, \\ Yoshiaki Kitamura ${ }^{1}$, Koichi Tomita ${ }^{4}$ and Noriaki Takeda ${ }^{1}$ \\ 1 Department of Otolaryngology, Graduate School of Biomedical Sciences, University of Tokushima, \\ Tokushima 770-8503, Japan; go-sato@tokushima-u.ac.jp (G.S.); momomonga_buchi@yahoo.co.jp (M.M.); \\ azuma.takahiro@tokushima-u.ac.jp (T.A.); ykitamura@tokushima-u.ac.jp (Y.K.); \\ takeda@tokushima-u.ac.jp (N.T.) \\ 2 Department of Otolaryngology, Tokushima Prefectural Central Hospital, Tokushima 770-8503, Japan; \\ matsudakazunori1024@gmail.com \\ 3 Department of Otolaryngology Head and Neck Surgery, Nara Medical University, Kashihara 634-8522, Japan; \\ tkitahara@naramed-u.ac.jp \\ 4 Department of Anatomy and Developmental Neurobiology, Institute of Biomedical Science, Graduate School, \\ Tokushima University, Tokushima 770-8503, Japan; ktomita@tokushima-u.ac.jp \\ * Correspondence: fukudaj5@gmail.com
}

check for

updates

Citation: Fukuda, J.; Matsuda, K.; Sato, G.; Kitahara, T.; Matsuoka, M.; Azuma, T.; Kitamura, Y.; Tomita, K.; Takeda, N. Effects of Betahistine on the Development of Vestibular Compensation after Unilateral Labyrinthectomy in Rats. Brain Sci. 2021, 11, 360. https://doi.org/ $10.3390 /$ brainsci11030360

Academic Editor: Werner M. Graf

Received: 25 January 2021

Accepted: 8 March 2021

Published: 11 March 2021

Publisher's Note: MDPI stays neutral with regard to jurisdictional claims in published maps and institutional affiliations.

Copyright: (C) 2021 by the authors. Licensee MDPI, Basel, Switzerland. This article is an open access article distributed under the terms and conditions of the Creative Commons Attribution (CC BY) license (https:/ / creativecommons.org/licenses/by/ $4.0 /)$.

\begin{abstract}
Background: Vestibular compensation (VC) after unilateral labyrinthectomy (UL) consists of the initial and late processes. These processes can be evaluated based on the decline in the frequency of spontaneous nystagmus (SN) and the number of MK801-induced Fos-positive neurons in the contralateral medial vestibular nucleus (contra-MVe) in rats. Histamine H3 receptors (H3R) are reported to be involved in the development of VC. Objective: We examined the effects of betahistine, an H3R antagonist, on the initial and late processes of VC in UL rats. Methods: Betahistine dihydrochloride was continuously administered to the UL rats at doses of 100 and $200 \mathrm{mg} / \mathrm{kg} /$ day using an osmotic minipump. MK801 (1.0 mg/ kg) was intraperitoneally administered on days 7, 10, 12, and 14 after UL, while Fos-positive neurons were immunohistochemically stained in the contra-MVe. Results: The SN disappeared after $42 \mathrm{~h}$, and continuous infusion of betahistine did not change the decline in the frequency of SN. The number of MK801-induced Fos-positive neurons in contra-MVe significantly decreased on days 7, 10, and 12 after UL in a dose-dependent manner in the betahistine-treated rats, more so than in the saline-treated rats. Conclusion: These findings suggest that betahistine facilitated the late, but not the initial, process of VC in UL rats.
\end{abstract}

Keywords: vestibular compensation; betahistine dihydrochloride; medial vestibular nucleus; Fos; histamine $\mathrm{H} 3$ receptor

\section{Introduction}

Unilateral vestibular dysfunction causes spontaneous nystagmus (SN), changes in postural control, and locomotor deviation. SN is a high-frequency, mainly horizontal, spontaneous ocular nystagmus, with its quick phase directed to the unaffected side. However, the nystagmus and balance disorders recover gradually after the occurrence of the lesion. This functional restoration-based on the plasticity of the central vestibular system-is called vestibular compensation (VC) [1]. The VC is divided into two phases: static and dynamic. Static VC is further divided into the initial and late processes [2]. Unilateral labyrinthectomy (UL) causes a loss of resting activity in many neurons in the ipsilateral medial vestibular nucleus (ipsi-MVe), resulting in an imbalance in neural activity between the vestibular nuclei on each side of the brainstem. This imbalance in vestibular nuclear activities after UL induces SN. During the initial process of VC after UL, the suppression of the contralateral medial vestibular nucleus (contra-MVe) by the vestibular cerebellumvestibular nucleus inhibitory system equalizes the imbalance in vestibular nuclear activities. 
Consequently, the SN gradually decreases [2,3]. During the late process of VC after UL, the spontaneous firing of ipsi-MVe neurons is restored by changes in cell membrane properties, reinforcing the balance in neural activity between the vestibular nuclei on each side. Consequently, the neural activities between the vestibular nuclei on each side are balanced without the vestibular-cerebellum-induced suppression of the contra-MVe during the initial VC process $[4,5]$.

Betahistine is a histamine $\mathrm{H} 3$ receptor (H3R) antagonist and a partial histamine $\mathrm{H} 1$ receptor agonist. Betahistine is clinically used for the treatment of vertigo and Meniere's disease-like symptoms, and its anti-vertiginous action has been considered to be in part due to its increase of inner ear microcirculation via H1R. A previous study reported that betahistine accelerated the disappearance of SN after UL in cats, suggesting that betahistine facilitates VC after UL via H3R [6]. The aim of the present study was to investigate the effects of betahistine on the initial and late processes of $\mathrm{VC}$, using unilaterally labyrinthectomized rats (UL rats) as model animals. To evaluate the effects of betahistine on the initial process of VC in UL rats, we measured the decline in the frequency of $\mathrm{SN}$ after UL [7]. The late process of VC in UL rats can be evaluated by measuring the decline in the number of Fos-positive neurons being expressed in the contra-MVe after induction by MK801, an $N$-methyl-D-aspartate (NMDA) receptor antagonist [8]. Therefore, we investigated the effects of betahistine on the number of MK801-induced Fos-positive neurons in the contra-MVe of UL rats.

\section{Materials and Methods}

\subsection{Experimental Animals}

Experiments were performed using adult male Wistar rats (Japan SLC, Inc., Hamamatsu City, Shizouka, Japan) weighing approximately 150-200 g. The rats were individually housed in polycarbonate cages with wood chip bedding at an environmental temperature of $20-22{ }^{\circ} \mathrm{C}$ under a $12 \mathrm{~h} \mathrm{light/dark} \mathrm{cycle} \mathrm{(lights} \mathrm{on} \mathrm{at} \mathrm{08:00,} \mathrm{lights} \mathrm{off} \mathrm{at} \mathrm{20:00),} \mathrm{and} \mathrm{were}$ provided unlimited access to water and food. This study was conducted in accordance with the Fundamental Guidelines for Proper Conduct of Animal Experiments and Related Activities in Academic Research Institutions under the jurisdiction of the Ministry of Education, Culture, Sports, Science, and Technology, Japan. All procedures were approved by the Division for Animal Research Resources and Genetic Engineering Support Center for Advanced Medical Sciences, Institute of Biomedical Sciences, Tokushima University Graduate School (Animal Experiment Plan Approval No. T2019-9).

\subsection{Unilateral Labyrinthectomy}

The animals were anesthetized with isoflurane (Wako Pure Chemical Industries, Ltd., Osaka City, Osaka, Japan). The UL of the right ear was performed as previously described [9]. In brief, the tympanic bulla was opened under an operating microscope. The tympanic membrane, incus, and malleus were subsequently removed using a postauricular approach. The foot plate of the stapes was removed to open the oval window. The membranous labyrinth was destroyed by injecting $100 \%$ ethanol. An antibiotic (ofloxacin) cream was applied to the opened tympanic cavity to prevent infection at the end of surgery. Finally, the incision was closed, after which the animals were allowed to recover in light. UL was confirmed by the appearance of $\mathrm{SN}$ and postural deviation after recovery from anesthesia. The sham-operated rats were subjected to only a right postauricular skin incision under anesthesia.

\subsection{Drug Administration}

MK801 (Santa Cruz Biotechnology, Inc., Santa Cruz, CA, USA) was dissolved in 0.9\% saline and injected intraperitoneally at a dose of $1.0 \mathrm{mg} / \mathrm{kg}$ in rats $7,10,12$, and 14 days after UL. The MK801 dose of $1.0 \mathrm{mg} / \mathrm{kg}$ was used as it has been reported to induce complete decompensation in UL rats [2]. 
Betahistine dihydrochloride (Sigma-Aldrich, St. Louis, MO, USA) was dissolved in $0.9 \%$ saline and infused intraperitoneally $(0.5 \mu \mathrm{L} / \mathrm{h})$ at a dose of $100 \mathrm{mg} / \mathrm{kg} /$ day or $200 \mathrm{mg} / \mathrm{kg} /$ day using an osmotic minipump (Alzet, Palo Alto, CA, USA) immediately after UL. The osmotic minipump was filled with either saline or betahistine dihydrochloride and implanted intraperitoneally until day 14 after UL. These doses were chosen because betahistine dihydrochloride administered at a dose of $100 \mathrm{mg} / \mathrm{kg} /$ day has been reported to induce significant improvements in posture recovery in cats after UL [10].

\subsection{Behavioral Examination}

The eye movements were recorded using a video camera (Sony HDR-CX500V, Sony Corporation, Minato City, Tokyo, Japan) with a zoom lens. The frequency of SN in UL rats was counted as the number of quick phase beats in $15 \mathrm{~s}$. The video images were recorded on a memory card and replayed on a liquid crystal display screen. The frequency of SN was counted three times for each rat, and the average value was calculated as previously described [8]. The values were measured at $0.5,1,2,3,6,12,18,24,30,36$, and $42 \mathrm{~h}$ after UL.

\subsection{Tissue Preparation and Immunohistochemical Staining}

Tissue preparation and immunohistochemical staining were performed as described previously [8]. The rats were deeply anesthetized with isoflurane $2 \mathrm{~h}$ after intraperitoneal administration of MK801. Following this, they were perfused transcardially with $100 \mathrm{~mL}$ of $4{ }^{\circ} \mathrm{C}$ saline and then with $250 \mathrm{~mL}$ of $4 \%$ paraformaldehyde in $0.1 \mathrm{~mol} / \mathrm{L}$ phosphate buffer $(\mathrm{PB})$. The rat brain was promptly extracted after perfusion-fixation and post-fixed in the same fixative solution at $4{ }^{\circ} \mathrm{C}$ for 1 day. The fixed brains were submerged in $30 \%$ sucrose $\mathrm{PB}$ at $4{ }^{\circ} \mathrm{C}$ for 2 days. The tissues were frozen and sectioned to a thickness of $30 \mu \mathrm{m}$ using a cryostat (Leica CM1850, Leica Biosystems, Wetzlar, Germany), then the immunohistochemical reaction was visualized using the peroxidase-antiperoxidase method. The sections were pretreated with $0.1 \% \mathrm{H}_{2} \mathrm{O}_{2}$ in $0.3 \%$ Triton X-100 in phosphatebuffered saline (PBS) for $30 \mathrm{~min}$ and then incubated in 5\% normal goat serum (NGS) in PBS containing $0.3 \%$ Triton-X (PBST). The sections were then incubated with rabbit polyclonal anti-Fos antibody (Santa Cruz Biotechnology) diluted to 1:5000 in PBST containing 1\% NGS for 2 days at $4{ }^{\circ} \mathrm{C}$. Following incubation with the primary antibody and a brief wash, the sections were incubated with anti-rabbit IgG (Medical and Biological Laboratories Co., Ltd., Nagoya, Japan) diluted to 1:1000 in PBST for $1 \mathrm{~h}$ at room temperature. Fos expression in neurons was induced by incubating sections with a 3,3'-diaminobenzidine (DAB) Substrate Kit (Vector Laboratories, Burlingame, CA, USA).

\subsection{Cell Counting}

Transverse 30- $\mu \mathrm{m}$-thick sections of the brainstem were observed under bright-field microscopy at $40 \times$ and $100 \times$ magnification to detect Fos-positive neurons in the contraMVe. Only cells that had significant levels of DAB reaction product in their nuclei (i.e., above tissue background levels) were counted using a digital image analysis system (ImageJ ver. 1.52a software).

\subsection{Experimental Protocol}

Depending on the operative and postoperative treatments, the experimental rats were divided into four groups: (1) rats that received saline after UL, (2) rats that received betahistine dihydrochloride (100 mg/ kg/day) after UL, (3) rats that received betahistine dihydrochloride ( $200 \mathrm{mg} / \mathrm{kg} /$ day) after UL, and (4) sham-operated rats. Rats that received saline were subjected to UL of the right ear and received intraperitoneal injection of saline with an osmotic minipump $(n=19)$. Then, they were intraperitoneally administrated a single dose of MK801 $(1.0 \mathrm{mg} / \mathrm{kg})$ on days $7(\mathrm{n}=4), 10(\mathrm{n}=5), 12(\mathrm{n}=5)$, and $14(\mathrm{n}=5)$ after UL.

Rats that received betahistine dihydrochloride were subjected to UL of the right ear and received intraperitoneal infusion of betahistine dihydrochloride at a dose of 
$100 \mathrm{mg} / \mathrm{kg} /$ day $(\mathrm{n}=21)$ or $200 \mathrm{mg} / \mathrm{kg} /$ day $(\mathrm{n}=20)$ with an osmotic minipump. Rats that received $100 \mathrm{mg} / \mathrm{kg} /$ day betahistine dihydrochloride were then intraperitoneally administered a single dose of MK801 $(1.0 \mathrm{mg} / \mathrm{kg})$ on days $7(\mathrm{n}=6), 10(\mathrm{n}=7), 12(\mathrm{n}=4)$, and $14(\mathrm{n}=4)$ after UL. Rats that received $100 \mathrm{mg} / \mathrm{kg} /$ day betahistine dihydrochloride were also intraperitoneally administered a single dose of MK801 $(1.0 \mathrm{mg} / \mathrm{kg})$ on days $7(n=4), 10(n=7), 12(n=4)$, and $14(n=5)$ after UL. The sham-operated rats were intraperitoneally administered a single dose of MK801 $(1.0 \mathrm{mg} / \mathrm{kg})$ on day 14 after the sham operation $(n=4)$.

\subsection{Statistical Analysis}

The frequency of $\mathrm{SN}$ and the number of Fos-positive neurons were analyzed using a two-factorial analysis of variance followed by the Shceffé's test. Statistical significance was set at $p<0.05$.

\section{Results}

The frequency of SN was measured $0.5,1,2,3,6,12,18,24,30,36$, and $42 \mathrm{~h}$ after UL (Figure 1). At each time point, the SN frequency values were not significantly different between the UL rats that received betahistine (100 or $200 \mathrm{mg} / \mathrm{kg} /$ day) and those that received saline. The $\mathrm{SN}$ disappeared at $42 \mathrm{~h}$ in all $\mathrm{UL}$ rats that received either betahistine or saline.

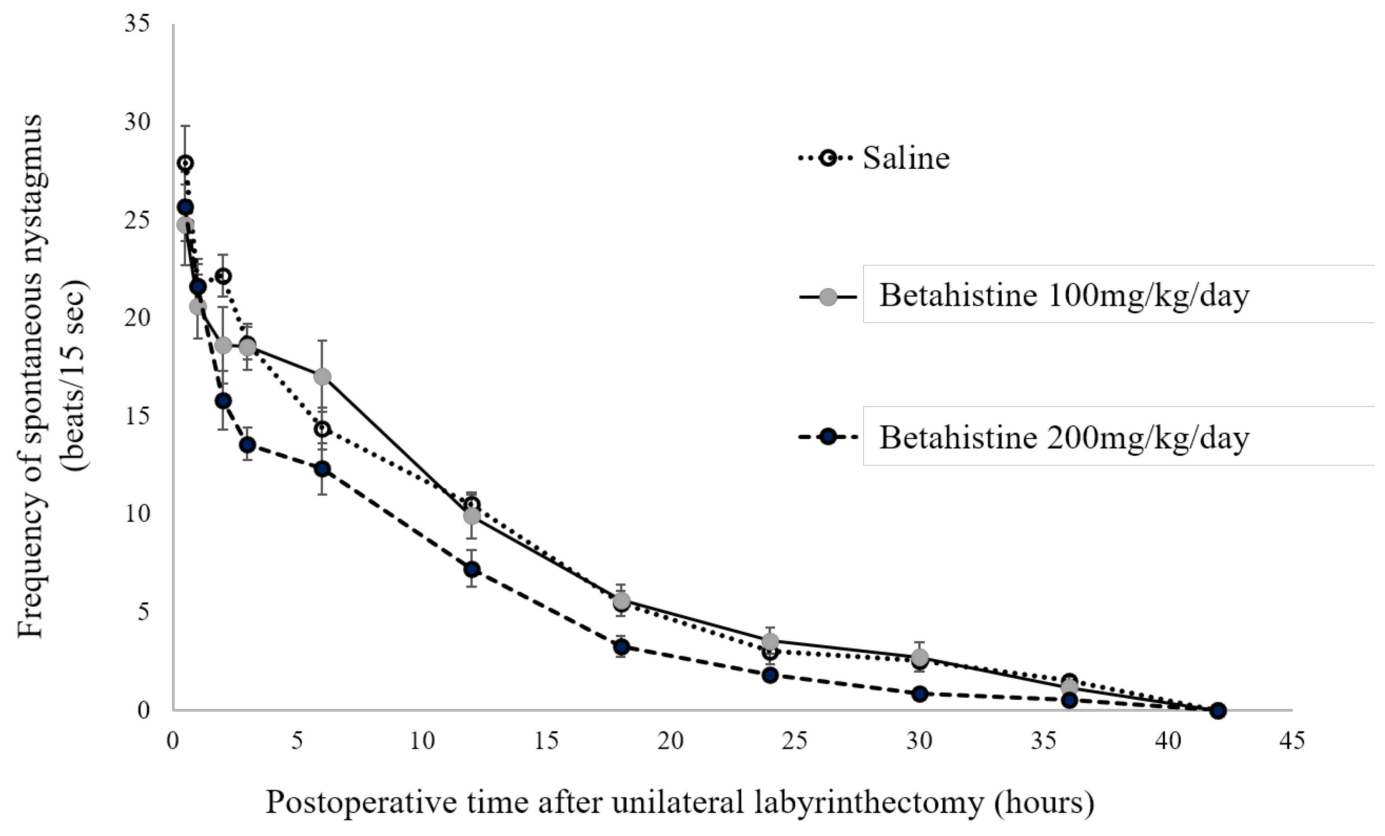

Figure 1. Effects of betahistine on changes with time in the frequency of spontaneous nystagmus after unilateral labyrinthectomy in rats. Each point represents the mean number of quick-phase eye movements in 15 s. Saline: rats received intraperitoneal infusion of saline, shown as open circles with a dotted line. Betahistine $100 \mathrm{mg} / \mathrm{kg} / \mathrm{day}$ : rats received intraperitoneal infusion of betahistine at a dose of $100 \mathrm{mg} / \mathrm{kg} /$ day, shown as gray circles with a solid line. Betahistine $200 \mathrm{mg} / \mathrm{kg} /$ day: rats received intraperitoneal infusion of betahistine at a dose of $200 \mathrm{mg} / \mathrm{kg} /$ day, shown as filled circles with a broken line.

MK801 induced the appearance of Fos-positive neurons in the contra-MVe in UL rats. Figure 2 shows the substantial number of MK801-induced Fos-positive neurons in the contra-MVe on day 10 after UL in saline-treated rats. In rats that received saline, the number of MK801-induced Fos-positive neurons in the contra-MVe gradually decreased and equaled the number in the sham-operated rats on day 14 after UL (Figure 3). The number of MK801-induced Fos-positive neurons in the $100 \mathrm{mg} / \mathrm{kg} /$ day betahistine-treated rats was significantly lower than that in the saline-treated UL rats on days 7, 10, and 12 
(Figure 3), and equaled the number in the sham-operated rats on day 12 after UL. The number of MK801-induced Fos-positive neurons in the $200 \mathrm{mg} / \mathrm{kg}$ /day betahistine-treated rats was also significantly lower than that in the saline-treated UL rats on days 7, 10, and 12 , but equaled the number in the sham-operated rats on day 10 after UL. Moreover, the number of MK801-induced Fos-positive neurons on day 10 after UL was significantly lower in the rats that received $200 \mathrm{mg} / \mathrm{kg} /$ day betahistine than in those that received a $100 \mathrm{mg} / \mathrm{kg} /$ day dose (Figure 3).

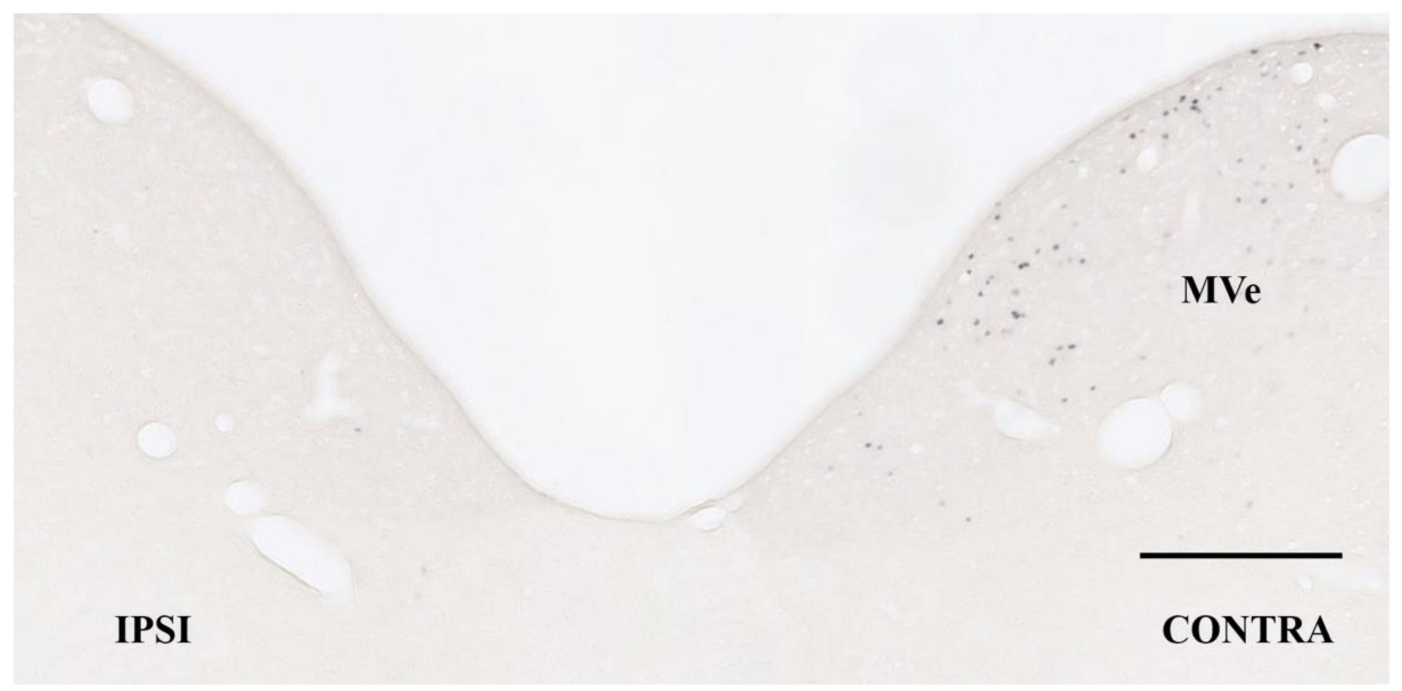

Figure 2. Bright-field micrograph of Fos-positive neurons in the contralateral medial vestibular nucleus $2 \mathrm{~h}$ after administration of MK801 in unilateral labyrinthectomized rats who received continuous intraperitoneal administration of saline on post-operative day 10 after unilateral labyrinthectomy (UL). Bar: $200 \mu \mathrm{m}$. MVe: medial vestibular nucleus; IPSI: ipsilateral to UL; CONTRA: contralateral to UL.

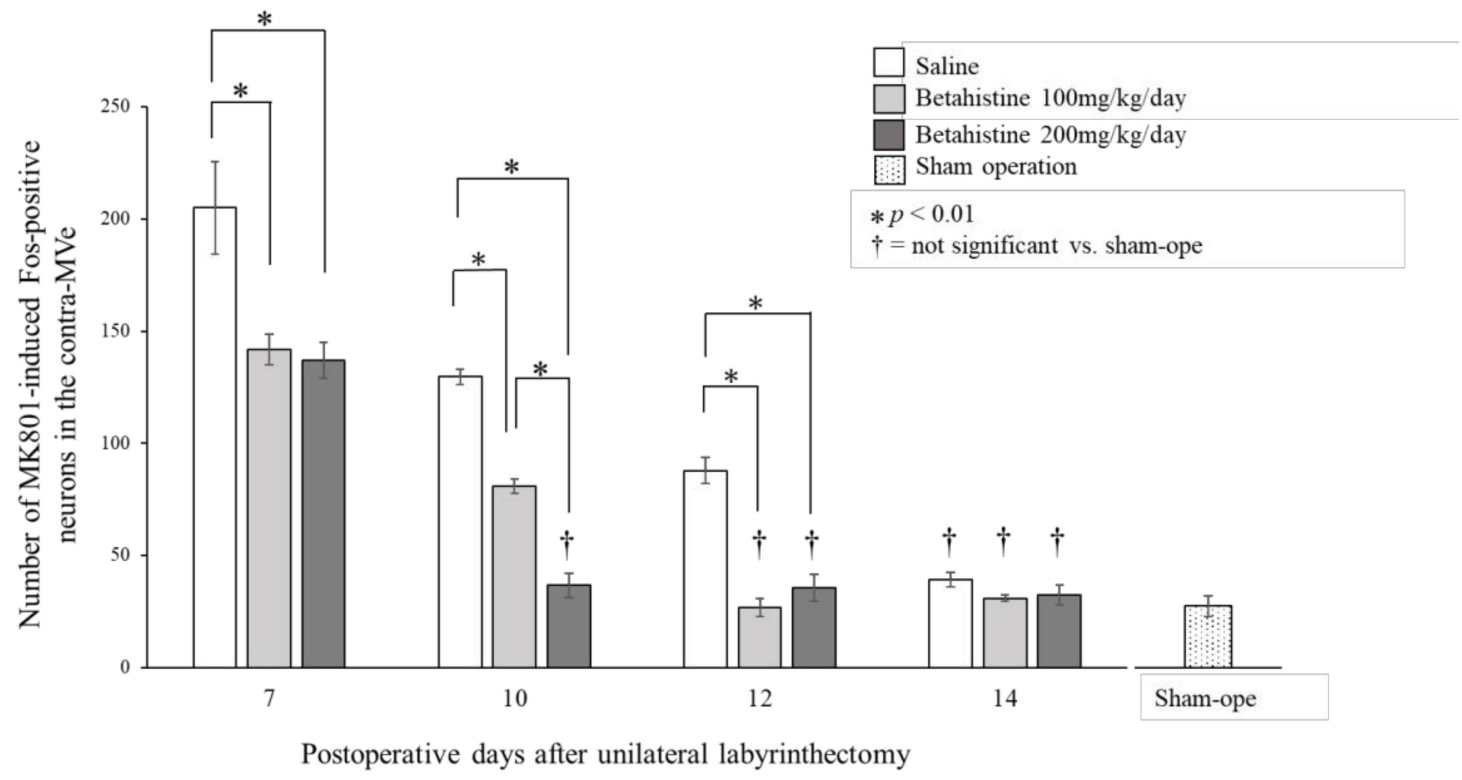

Figure 3. Effects of betahistine on changes with time in the number of Fos-positive neurons in the contralateral medial vestibular nucleus $2 \mathrm{~h}$ after administration of MK801 in unilateral labyrinthectomized rats. Saline: rats received intraperitoneal infusion of saline, shown as open columns. Betahistine $100 \mathrm{mg} / \mathrm{kg} /$ day: rats received intraperitoneal infusion of betahistine at a dose of $100 \mathrm{mg} / \mathrm{kg}$ / day, shown as gray columns. Betahistine $200 \mathrm{mg} / \mathrm{kg}$ /day: rats received intraperitoneal infusion of betahistine at a dose of $200 \mathrm{mg} / \mathrm{kg}$ /day, shown as filled columns. Sham operation (sham-ope): rats received sham operation only. 


\section{Discussion}

In the present study, the decline in the frequency of SN after UL was not different between the betahistine- and saline-treated rats. In both groups, the SN disappeared $42 \mathrm{~h}$ after UL. Because the decline in the frequency of SN after UL indicates the development of the initial process of $\mathrm{VC}$ in rats [7], our findings suggest that betahistine did not affect this process of VC after UL in these rats.

A previous study reported that the disappearance of SN after UL was accelerated in cats that received betahistine transorally [6]. A recent study reported that betahistine microinjected into the bilateral medial vestibular nuclei also decreased the frequency of SN in the UL rats more so than in the control rats [11]. However, our previous study showed that intraperitoneal infusion of thioperamide (another H3R antagonist) did not change the frequency of SN after UL in rats [8]. Moreover, intraperitoneal injection of thioperamide has been reported to delay the recovery of body tilt after UL in goldfish [12]. Thus, the effects of H3R antagonists-including betahistine- on the initial process of VC varies with species and administration methods.

In the present study, the number of MK801-induced Fos-positive neurons in the contra-MVe of betahistine-treated UL rats decreased in a dose-dependent manner and was lower than that in the saline-treated UL rats. The numbers of such neurons in the salinetreated, $100 \mathrm{mg} / \mathrm{kg} /$ day betahistine-treated, and $200 \mathrm{mg} / \mathrm{kg} /$ day betahistine-treated UL rats equaled those in the sham-operated rats on days 14,12, and 10 after UL, respectively. As the decline in the number of MK801-induced Fos-positive neurons in the contra-MVe after UL indicates the development of the late process of VC in rats [8], these findings suggest that betahistine facilitates the late process of VC after UL in rats in a dose-dependent manner.

Because Fos is the protein product of an immediate early gene, c-Fos is a marker of neural activity [13]. It is assumed that the MK801-induced Fos-positive neurons in the contra-MVe are suppressed by the NMDA receptor-mediated vestibular cerebellumvestibular nucleus inhibitory system in the initial process of VC after UL [14]. Our previous study showed that the MK801-induced Fos-positive neurons in the contra-MVe disappeared in accordance with the recovery of the spontaneous firing of the ipsi-MVe neurons in the late process of VC after UL [8]. Therefore, the present findings further suggest that betahistine facilitates recovery of the spontaneous firing of the ipsi-MVe neurons in UL rats.

H3Rs are located in the presynaptic histaminergic fibers and act as inhibitory autoreceptors [15]. The histaminergic neurons in the tuberomammillary nuclei of the posterior hypothalamus give rise to the axons to the MVe [16]. Therefore, it is assumed that H3R antagonists increase the release of histamine in the MVe. Indeed, betahistine, an H3R antagonist, has been reported to upregulate the gene expression of histidine decarboxylase, a histamine-synthesizing enzyme in the tuberomammillary nuclei of cats, suggesting that betahistine upregulates histamine turnover and release [17]. Moreover, histamine H1, $\mathrm{H} 2$, and $\mathrm{H} 3$ receptors $(\mathrm{H} 1 \mathrm{R}, \mathrm{H} 2 \mathrm{R}$, and $\mathrm{H} 3 \mathrm{R})$ are also expressed in the MVe of rats [3,18], while H1R- and H2R-mediated histamine cause excitation in the MVe neurons in vivo and in vitro [19-21]. Therefore, it is suggested that the H3R antagonist acts by increasing histamine release and causing excitability in the MVe neurons. Betahistine works as both a partial H1R agonist and a more potent H3R antagonist [22,23], while H1R expression increases in a restricted manner in the ipsi-MVe neurons of UL rats [11]. Therefore, it is suggested that the betahistine-induced release of histamine causes the activation of the MVe neurons and facilitates the recovery of the spontaneous firing of the ipsi-MVe neurons. It is further suggested that the neural activities between the vestibular nuclei on each side are finally balanced in the late VC process, without the vestibular cerebellum-induced suppression of the contra-MVe that occurs during the initial VC process. Consequently, the late $\mathrm{VC}$ process was facilitated in the UL rats that received betahistine.

Clinically, betahistine is usually used as an anti-vertiginous drug for the treatment of acute and subacute stages of vertigo. Betahistine has been reported to improve the recovery of static vestibular symptoms in patients with Meniere's disease after unilateral vestibular nerve transection [24]. This clinical finding suggests that betahistine may also 
facilitate the development of VC after unilateral vestibular deficits in humans. Based on the present finding using betahistine at doses of $100-200 \mathrm{mg} / \mathrm{kg} /$ day in rats and previous reports using betahistine at doses of $50-100 \mathrm{mg} / \mathrm{kg} /$ day in cats [10], our clinical goal is to prove the hypothesis that a high dose of betahistine facilitates the process of VC in patients with unilateral vestibular deficits, who complain of refractory dizziness due to delays in development of VC. In fact, our preliminary study showed long-term administration of betahistine at a high dose of $72 \mathrm{mg} /$ day for 16 weeks improved Dizziness Handicap Inventory scores in refractory dizzy patients with vestibular neuritis. Although the original daily maximum dose of betahistine was $48 \mathrm{mg}$, it was recently reported that long-term administration for 12 months with betahistine at higher dose, such as $144 \mathrm{mg} /$ day and more recently $480 \mathrm{mg} /$ day, was effective for the treatment of Meniere's disease [25,26]. Further studies are needed on whether log-term treatment with a high dose of betahistine facilitates the process of $\mathrm{VC}$ in refractory dizzy patients with unilateral vestibular deficits.

\section{Conclusions}

In the present study, we evaluated the effects of betahistine on the initial and late processes of VC in UL rats by measuring the decrease in the frequency of $\mathrm{SN}$ and the decline in the number of MK801-induced Fos-positive neurons in the contra-MV, respectively. Betahistine did not affect the initial VC process, but facilitated the development of the late VC process in UL rats. Our results suggest that betahistine blocks presynaptic H3R and promotes histamine release. This, in turn, activates the MVe neurons via H1Rs and H2Rs to facilitate the recovery of the spontaneous firing of the ipsi-MVe neurons, accelerating the late process of VC after UL in rats. The facilitatory effect of betahistine on VC is an underlying mechanism in the management of acute and subacute vertigo in patients with unilateral vestibular disorders.

Author Contributions: Conceptualization, J.F., K.M., G.S., T.K., M.M., T.A., Y.K., and N.T.; methodology, J.F., K.M., T.K., and K.T.; investigation, J.F., and K.M.; formal analysis, J.F., K.M., and N.T.; data curation, J.F.; writing — original draft preparation, J.F.; writing—review and editing, J.F., K.M., G.S., T.K., and N.T.; visualization, J.F.; supervision, N.T.; project administration, N.T.; funding acquisition, K.M. All authors have read and agreed to the published version of the manuscript.

Funding: This work was supported by JSPS KAKENHI Grant Number JP19K09911, a Grant-in-Aid for basic research (C).

Institutional Review Board Statement: The study was conducted according to the guidelines of the Declaration of Helsinki, and approved by the Ethics Committee of the Division for Animal Research Resources and Genetic Engineering Support Center for Advanced Medical Sciences, Institute of Biomedical Sciences, Tokushima University Graduate School (protocol code T2019-9 and date of approval is 14 May 2019).

Conflicts of Interest: The authors declare no conflict of interest in this study.

\section{References}

1. Dutia, M.B. Mechanisms of vestibular compensation: Recent advances. Curr. Opin. Otolaryngol. Head Neck Surg. 2010, 18, 420-424. [CrossRef] [PubMed]

2. Kitahara, T.; Takeda, N.; Kiyama, H.; Kubo, T. Molecular mechanisms of vestibular compensation in the central vestibular system-review. Acta Otolaryngol. Suppl. 1998, 539, 19-27. [CrossRef] [PubMed]

3. Pillot, C.; Heron, A.; Cochois, V.; Tardivel-Lacombe, J.; Ligneau, X.; Schwartz, J.C.; Arrang, J.M. A detailed mapping of the histamine $\mathrm{H}(3)$ receptor and its gene transcripts in rat brain. Neuroscience 2002, 114, 173-193. [CrossRef]

4. Cameron, S.A.; Dutia, M.B. Cellular basis of vestibular compensation: Changes in intrinsic excitability of MVN neurons. Neuroreport 1997, 8, 2595-2599. [CrossRef]

5. Beraneck, M.; Hachemaoui, M.; Idoux, E.; Ris, L.; Uno, A.; Godaux, E.; Vidal, P.P.; Moore, L.E.; Vibert, N. Long-Term Plasticity of Ipsilesional Medial Vestibular Nucleus Neurons After Unilateral Labyrinthectomy. J. Neurophsiol. 2003, 90, 184-203. [CrossRef]

6. Tighilet, B.; Mourre, C.; Trottier, S.; Lacour, M. Histaminergic ligands improve vestibular compensation in the cat: Behavioural, neurochemical and molecular evidence. Eur. J. Pharmacol. 2007, 568, 149-163. [CrossRef] [PubMed]

7. Smith, P.F.; Darlington, C.L. Neurochemical mechanisms of recovery from peripheral vestibular lesions (vestibular compensation). Brain Res. Rev. 1998, 16, 19-27. [CrossRef] 
8. Matsuda, K.; Kitahara, T.; Ito, T.; Fukushima, M.; Fukuda, J.; Sato, G.; Kitamura, Y.; Abe, K.; Uno, A.; Tomita, K.; et al. A new immunohistochemical method to evaluate the development of vestibular compensation after unilateral labyrinthectomy in rats. Acta OtoLaryngol. 2019, 139, 505-510. [CrossRef]

9. Kitahara, T.; Takeda, N.; Saika, T.; Kudo, T.; Kiyama, H. Effects of MK801 on Fos expression in the rat brainstem after unilateral labyrinthectomy. Brain Res. 1995, 700, 182-190. [CrossRef]

10. Tighlet, B.; Leonard, J.; Lacour, M. Betahistine dihydrochloride treatment facilitates vestibular compensation in the cat. J. Vestib Res. 1995, 5, 53-66. [CrossRef]

11. Chen, Z.-P.; Zhang, X.-Y.; Peng, S.-Y.; Yang, Z.-Q.; Wang, Y.-B.; Zhang, Y.-X.; Chen, X.; Wang, J.-J.; Zhu, J.-N. Histamine H1 Receptor Contributes to Vestibular Compensation. J. Neurosci. 2019, 39, 420-433. [CrossRef]

12. Piratello, A.C.; Mattioli, R. Thioperamide delays vestibular compensation in goldfish. Neurosci. Lett. 2007, 415, 146-148. [CrossRef] [PubMed]

13. Cirelli, C.; Pompeiano, M.; D’Ascanio, P.; Arrighi, P.; Pompeiano, O. c-fos Expression in the rat brain after unilateral labyrinthectomy and its relation to the uncompensated and compensated stages. Neuroscience 1996, 70, 515-546. [CrossRef]

14. Kitahara, T.; Fukushima, M.; Takeda, N.; Saika, T.; Kubo, T. Effects of Pre-flocculectomy on Fos Expression and NMDA Receptormediated Neural Circuits in the Central Vestibular System after Unilateral Labyrinthectomy. Acta Otolaryngol. 2000, 120, 866-871. [PubMed]

15. Arrang, J.M.; Garbarg, M.; Schwartz, J.C. Auto-inhibition of brain histamine release mediated by a novel class $\left(\mathrm{H}_{3}\right)$ of histamine receptor. Nature 1983, 302, 832-837. [CrossRef]

16. Takeda, N.; Morita, M.; Kubo, T.; Yamatodani, A.; Wada, H.; Tohyama, M.; Matsunaga, T. Histaminergic projection from the posterior hypothalamus to the medial vestibular nucleus of rats and its relation to motion sickness. In The Vestibular System: Neurophysiological and Clinical Research; Graham, M.D., Kemink, J.L., Eds.; Raven Press: New York, NY, USA, 1987 ; pp. 571-580.

17. Tighilet, B.; Trottier, S.; Mourre, C.; Chotard, C.; Lacour, M. Betahistine dihydrochloride interaction with the histaminergic system in the cat: Neurochemical and molecular mechanisms. Eur. J. Pharmacol. 2002, 446, 63-73. [CrossRef]

18. Zhow, L.; Zhow, W.; Zhang, S.; Liu, B.; Leng, Y.; Zhou, R.; Kong, W. Changes in Histamine Receptors (H1, H2, and H3) Expression in Rat Medial Vestibular Nucleus and Flocculus after Unilateral Labyrinthectomy: Histamine Receptors in Vestibular Compensation. PLoS ONE 2013, 8, e66684. [CrossRef]

19. Serafin, M.; Khateb, A.; Vibert, N.; Mühlethaler, M. Medial vestibular nucleus in the guinea-pig: Histaminergic receptors. I. An in vitro study. Exp. Brain Res. 1993, 93, 242-248. [CrossRef]

20. Wang, J.-J.; Dutia, M.B. Effects of histamine and berahistine on rat medial vestibular nucleus neurones: Possible mechanism of action of anti-histaminergic drugs in vertigo and motion sickness. Exp. Brain Res. 1995, 105, 18-24. [CrossRef]

21. Zhang, X.-Y.; Yu, L.; Zhuang, Q.-X.; Peng, S.-Y.; Zhu, J.-N.; Wang, J.-J. Postsynaptic mechanisms underlying the excitatory action of histamine on medial vestibular nucleus neurons in rats. Br. J. Pharmacol. 2013, 170, 156-169. [CrossRef] [PubMed]

22. Arrang, J.M.; Garbarg, M.; Quach, T.T.; Tuong, M.D.T.; Yeramian, E.; Schwartz, J.C. Action of betahistine at histamine receptors in the brain. Eur J. Pharmacol. 1985, 111, 73-84. [CrossRef]

23. Timmerman, H. Histamine agonists and antagonists. Acta Otolaryngol. Suppl. 1991, 479, 5-11. [CrossRef]

24. Redon, C.; Lopez, C.; Bernard-Demanze, L.; Dumitrescu, M.; Magnan, J.; Lacour, M.; Borel, L. Betahistine treatment improves the recovery of static symptoms in patients with unilateral vestibular loss. J. Clin. Pharmacol. 2011, 51, 538-548. [CrossRef] [PubMed]

25. Strupp, M.; Hupert, D.; Frenzel, C.; Wagner, J.; Hahn, A.; Jahn, K.; Zingler, V.C.; Mansmann, U.; Brandt, T. Long-term prophylactic treatment of attacks of vertigo in Meniere's disease-comparison of a high with a low dosage of betahistine in an open trial. Acta Otolaryngol. 2008, 128, 520-524. [CrossRef] [PubMed]

26. Lezius, F.; Adrion, C.; Mansmann, U.; Jahn, K.; Strupp, M. High-dosage betahistine dihydrochloride between 288 and 480 mg/day in patients with severe Meniere's disease: A case series. Eur Arch. Otorhinolaryngol. 2011, 268, 1237-1240. [CrossRef] 